\section{CCL2 and T cells in pulmonary fibrosis: an old player gets a new role}

\author{
Awo D Osafo-Addo, Erica L Herzog
}

Idiopathic pulmonary fibrosis (IPF) is an incurable condition characterised by progressive extracellular matrix deposition and tissue remodelling in the adult human lung. ${ }^{1}$ Because 5-year mortality rates approach $70 \%$, new approaches to treatment are sorely needed. IPF is thought to result from an abnormal wound healing response caused by an unknown insult to the lung epithelium that results in the recruitment and activation of myofibroblasts via incompletely understood mechanisms. ${ }^{1}$ The contribution of immune activation to these processes remains unknown. While the contribution of innate cell populations such as macrophages is gaining increased acceptance, the contribution of $\mathrm{T}$ cell driven adaptive immune responses remain controversial. ${ }^{2}$ The paper by Milger et $a l^{3}$ represents an important step forward in our understanding of this issue.

Historically, IPF has been defined as a non-immune entity due to its lack of an identifiable antigen driven immune response, lack of inflammatory infiltrate on lung biopsy, lack of benefit from conventional immunosuppressive therapies and lack of a requirement for lymphocytes for development of maximal fibrosis in commonly used animal models. ${ }^{12}$ However, the now seminal finding that lymphocyte modulating agents actually worsen outcomes in IPF $^{4}$ combined with human and animal data demonstrating an imbalance between $\mathrm{T}$ helper populations in these settings suggests a critical role for lymphocytes in the orchestration of fibrotic responses. For instance, Th2 and Th17 cells are associated with fibrogenesis, while Th1, Th22 and $\gamma \delta$-T cells appear to be at least partially protective. ${ }^{5-9}$ In contrast, regulatory $\mathrm{T}$ cells (Tregs) can differentially promote or suppress fibrosis depending on the setting. ${ }^{10}$ When viewed in combination, these data suggest a cell-specific and state-specific contribution of $\mathrm{T}$ lymphocytes to IPF, leading to the growing recognition of $\mathrm{T}$ cell heterogeneity as an

Department of Internal Medicine, Yale University School of Medicine, New Haven, Connecticut, USA

Correspondence to Professor Erica L Herzog, Department of Internal Medicine, Yale University School of Medicine, 333 Cedar St, New Haven, CT 06510, USA; erica.herzog@yale.edu important area of investigation for this incurable disease.

CCL2 is a chemokine which orchestrates immune cell recruitment via its preferential binding to the CCR2 receptor. ${ }^{11} \mathrm{It}$ is induced during inflammatory responses when immune cells are required for tissue repair. ${ }^{11}$ Both human and murine studies have shown that CCL2 contributes to fibrosis through a variety of mechanisms involving inflammation, angiogenesis and myofibroblast accumulation. ${ }^{12}$ Specifically, elevations in CCL2 have been found in the serum, ${ }^{13}$ bronchoalveolar lavage ${ }^{14}$ and the alveolar epithelium ${ }^{15}$ of patients with IPF, and deletion or inhibition of CCR2 reduces pulmonary fibrosis in several experimental settings. ${ }^{16}$ Furthermore, studies using murine models of pulmonary fibrosis and primary IPF lung fibroblasts suggest an interplay between TGF $\beta 1$, interleukin (IL)13 and CCL2 promotes fibroproliferation ${ }^{17} 18$ and excessive activation of the CCR2/CCL2 axis is predictive of poor prognosis in patients with IPF. $^{19}$

Based on this preclinical evidence, Carlumab (a humanised monoclonal antibody targeting CCL2) was tested for efficacy in a randomised controlled trial of patients with IPF. ${ }^{12}$ This study, which was published in 2015, failed to find a benefit for Carlumab therapy and in fact was terminated early due to a trend towards worsening of lung function in one of the treatment arms. Now, for the first time, the study by Milger et $a l^{3}$ offers a possible explanation to the biology behind these observations.

Milger $e t a l^{3}$ identify CCR2 expressing $\mathrm{CD} 4+\mathrm{T}$ lymphocytes as a subset of CD4 + cells displaying anti-inflammatory and antifibrotic properties and explore their role in lung fibrosis. Specifically, studies performed in the bleomycin mouse model reveal the accumulation of CCR2 expressing CD4+ T cells $(\mathrm{CCR} 2+\mathrm{CD} 4+)$ in the fibrotic lung. The majority of these cells were phenotyped as effector memory $\mathrm{T}$ cells and demonstrated migratory potential due to their expression of homing receptors. Curiously, however, these cells also bear hallmarks of natural Tregs in that they express high levels of FoxP3 and IL-10. Functionally, CCR2 + CD4+ cells suppress effector
$\mathrm{T}$ cell proliferation in similar magnitude as natural Tregs and adoptive transfer of these cells improves fibrosis in bleomycin treated mice. In human correlates of these findings, increased concentrations of CCR2 + CD4 + cells are seen in bronchoalveolar lavage specimens from patients with IPF when compared with controls. Taken together, these data show CCL2 might act on these CCR2 + CD4+ cells to abrogate inflammation and fibrosis, and suggest that the therapeutic failure of Carlumab might be related to suppression of this population.

The impact of the study by Milger et $a l^{3}$ cannot be overstated. In addition to offering a potential explanation for the surprising failure of CCL2 targeted therapies, the work provides more global insight into IPF. For example, it supports the emerging concept that a single receptor such as CCR2 might play different roles depending on its temporospatial expression during injury and repair. It also suggests that CCL2 might play equal and opposing roles in innate and adaptive responses; specifically, to function as a recruiter of fibrosis-promoting macrophages while simultaneously inducing the accumulation of immunosuppressive Treg population. However, because CCR2 is also expressed on alveolar epithelium and fibroblasts, the effects of CCL2 neutralisation might extend to the regulation of injury and repair responses in stromal cells. It also demonstrates the need for improved modelling systems since the function of the CCR2 + CD4 + cells in the context of experimentally induced fibrosis in mice may not necessarily recapitulate the situation in human disease. The study also highlights the need for personalised and specific treatments that can selectively target detrimental cells of interest and supports the growing consensus that in the complex milieu of the fibrotic lung, interruption of a single receptor-ligand interaction may be insufficient to overcome the activation of multiple competing and compensatory pathways.

As with any novel finding, many questions remain. The relationship of these CCR2 + CD4 + cells to other lymphocyte populations, as well as to other immune cells present in the IPF lung, will require further investigation. The contribution of these cells to injury and remodelling remains undeciphered, though the generation of mice with inducible CCR2 deletion specifically targeted to CD4+ cells might answer this question. The relationship of CCR2+ CD4+ cells to the IPF disease state remains unelucidated, as does their relationship with disease progression 
and response to therapy. Nevertheless, the study by Milger et $a l^{3}$ firmly frames CCR $2+$ CD $4+$ cells as a new and exciting area of study of CCL2 biology as it relates to pulmonary fibrosis. Further understanding of these findings will advance the evolving understanding of the complex contribution of lymphocyte heterogeneity to IPF and related conditions affecting the adult mammalian lung.

Contributors ADO-A wrote the manuscript. ELH edited each draft and created the final version.

Competing interests None declared.

Provenance and peer review Commissioned; externally peer reviewed.

(C) Article author(s) (or their employer(s) unless otherwise stated in the text of the article) 2017. All rights reserved. No commercial use is permitted unless otherwise expressly granted.

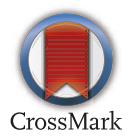

To cite Osafo-Addo AD, Herzog EL. Thorax 2017;72:967-968.

Published Online First 7 September 2017

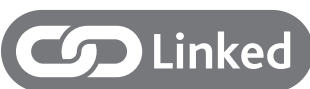

- http://dx.doi.org/10.1136/thoraxjnl-2016-208423
Thorax 2017;72:967-968.

doi:10.1136/thoraxjnl-2017-210517

\section{REFERENCES}

1 Blackwell TS, Tager AM, Borok Z, et al. Future directions in idiopathic pulmonary fibrosis research. An NHLBI workshop report. Am J Respir Crit Care Med 2014;189:214-22.

2 Crystal RG, Bitterman PB, Mossman B, et al. Future research directions in idiopathic pulmonary fibrosis: summary of a National Heart, Lung, and Blood Institute working group. Am J Respir Crit Care Med 2002;166:236-46.

3 Milger K, Yu Y, Brudy E, et al. Pulmonary CCR2(+) CD4(+) $T$ cells are immune regulatory and attenuate lung fibrosis development. Thorax 2017;72:1007-20.

4 Raghu G, Anstrom KJ, King TE, et al. Prednisone, azathioprine, and $\mathrm{N}$-acetylcysteine for pulmonary fibrosis. N Engl I Med 2012;366:1968-77.

5 Saito A, Okazaki H, Sugawara I, et al. Potential action of IL-4 and IL-13 as fibrogenic factors on lung fibroblasts in vitro. Int Arch Allergy Immunol 2003:132:168-76.

6 Wilson MS, Madala SK, Ramalingam TR, et al. Bleomycin and IL-1 beta-mediated pulmonary fibrosis is IL-17A dependent. J Exp Med 2010;207:535-52.

7 Wynn TA. Fibrotic disease and the $T(H) 1 / T(H) 2$ paradigm. Nat Rev Immunol 2004;4:583-94.

8 Pociask DA, Chen K, Choi SM, et al. $\gamma \delta$ T cells attenuate bleomycin-induced fibrosis through the production of CXCL10. Am J Pathol 2011;178:1167-76.

9 Keane MP, Belperio JA, Burdick MD, et al. IL-12 attenuates bleomycin-induced pulmonary fibrosis. Am J Physiol Lung Cell Mol Physiol 2001;281:L92-7.

10 Boveda-Ruiz D, D'Alessandro-Gabazza CN, Toda M, et al. Differential role of regulatory $T$ cells in early and late stages of pulmonary fibrosis. Immunobiology 2013;218:245-54.

11 Lim SY, Yuzhalin AE, Gordon-Weeks AN, et al. Targeting the CCL2-CCR2 signaling axis in cancer metastasis. Oncotarget 2016;7:28697-710.

12 Raghu G, Martinez FJ, Brown KK, et al. CC-chemokine ligand 2 inhibition in idiopathic pulmonary fibrosis: a phase 2 trial of carlumab. Eur Respir 」 2015;46:1740-50.

13 Baran CP, Opalek JM, McMaken S, et al. Important roles for macrophage colony-stimulating factor, $\mathrm{CC}$ chemokine ligand 2, and mononuclear phagocytes in the pathogenesis of pulmonary fibrosis. Am J Respir Crit Care Med 2007;176:78-89.

14 Suga M, lyonaga K, Ichiyasu H, et al. Clinical significance of MCP-1 levels in BALF and serum in patients with interstitial lung diseases. Eur Respir J 1999;14:376-82.

15 Mercer PF, Johns RH, Scotton CJ, et al. Pulmonary epithelium is a prominent source of proteinaseactivated receptor-1-inducible CCL2 in pulmonary fibrosis. Am J Respir Crit Care Med 2009;179:414-25.

16 Moore BB. Following the path of CCL2 from prostaglandins to periostin in lung fibrosis. Am J Respir Cell Mol Biol 2014;50:848-52.

17 Zhu Z, Ma B, Zheng T, et al. IL-13-induced chemokine responses in the lung: role of CCR2 in the pathogenesis of IL-13-induced inflammation and remodeling. J Immunol 2002;168:2953-62.

18 Murray LA, Argentieri RL, Farrell FX, et al. Hyperresponsiveness of IPF/UIP fibroblasts: interplay between TGFbeta1, IL-13 and CCL2. Int J Biochem Cell Biol 2008;40:2174-82.

19 Shinoda H, Tasaka S, Fujishima S, et al. Elevated CC chemokine level in bronchoalveolar lavage fluid is predictive of a poor outcome of idiopathic pulmonary fibrosis. Respiration 2009;78:285-92. 